\title{
An Inverse Model for Two-dimensional Reflectors With Scattering
}

\author{
Vì C.E. Kronberg ${ }^{1, *}$, Martijn J.H. Anthonissen ${ }^{1}$, Jan H.M. ten Thije Boonkkamp ${ }^{1}$, and Wilbert L. IJzerman ${ }^{1,2}$ \\ ${ }^{1}$ Department of Mathematics and Computer Science, Eindhoven University of Technology (TU/e), PO Box 513, \\ 5600 MB Eindhoven, The Netherlands \\ ${ }^{2}$ Signify Research, High Tech Campus 7, 5656 AE Eindhoven, The Netherlands
}

\begin{abstract}
We present a novel approach of modelling surface light scattering in the context of freeform optical design. Using energy conservation, we derive an integral relation between the scattered and specular distributions. This integral relation reduces to a convolution integral in the case of isotropic scattering in the plane of incidence for cylindrically and rotationally symmetric problems.
\end{abstract}

\section{Introduction}

Freeform reflector design answers the question of transforming a given source light distribution into a given target light distribution. A contemporary technique involves solving a Monge-Ampère equation for the shape of the reflector [1]. If the system is cylindrically or rotationally symmetric, the procedure may be performed in two dimensions and the resulting differential equation is significantly easier to solve [2, Ch. 2-3]. Irrespective of the symmetry of the problem, these approaches rely on the law of reflection from geometrical optics, and are thus only valid under the assumption of perfect specular reflectors. When surface scattering is present, this no longer holds. In order to compute specular reflectors using known methods, whilst taking into account light scattering, we propose a scheme of converting the prescribed diffuse target distribution into an intermediate specular distribution - see Sec. 2, followed by a specular reflector computation - see Sec. 3 and Sec. 4 for an outline of the procedure. Some results are presented in Sec. 5 .

\section{Scattering Model}

The derivations in this section are detailed in [3].

\subsection{Cylindrically Symmetric Reflectors}

Restricting our attention to scattering in the plane of incidence, consider Fig. 1 showing the cross-section of a cylindrically symmetric reflector $\mathbf{r}(\varphi)=u(\varphi) \hat{\mathbf{e}}_{r}$, where $u(\varphi)$ is the radius function and $\hat{\mathbf{e}}_{r}=(\cos (\varphi), \sin (\varphi))^{\top}$ is the radial unit vector in polar coordinates, and $\varphi \in(-\pi, \pi)$ is measured counter-clockwise with respect to the positive $x$-axis.

A source ray $\hat{\mathbf{s}}$ is emitted from the line-source in $O$ at an angle $\varphi$ and strikes the reflector at $\mathcal{P}$. The specular ray $\hat{\mathbf{t}}$ with angle $\psi \in(-\pi, \pi)$ (not shown, analogous to

*e-mail: s.kronberg@tue.nl $\gamma \in(-\pi, \pi)$ in the figure) is given by the familiar law of reflection $(\operatorname{LoR}), \hat{\mathbf{t}}=\hat{\mathbf{s}}-2(\hat{\mathbf{s}} \cdot \hat{\mathbf{n}}) \hat{\mathbf{n}}$, where $\hat{\mathbf{n}}$ is the unit normal at $\mathcal{P}$. By convention, we take $\hat{\mathbf{s}} \cdot \hat{\mathbf{n}}<0$, i.e., the normal points back towards the source. The scattered ray $\hat{\mathbf{u}}$ is rotated by a stochastic parameter $\alpha \in(-\pi, \pi)$ with respect to $\hat{\mathbf{t}}$, and its direction is denoted $\gamma \equiv \psi+\alpha$. That is,

$$
\hat{\mathbf{u}}=\mathbf{R}(\alpha) \hat{\mathbf{t}}, \quad \mathbf{R}(\alpha)=\left(\begin{array}{cc}
\cos (\alpha) & -\sin (\alpha) \\
\sin (\alpha) & \cos (\alpha)
\end{array}\right) .
$$

In terms of mappings, let $m(\varphi)=\psi$ be the law of reflection yielding the direction of $\hat{\mathbf{t}}, s(\psi ; \alpha)=\gamma$ be the socalled scattering map representing the direction of $\hat{\mathbf{u}}$, and $a(\psi, \gamma)=\alpha$ be a map yielding the stochastic parameter $\alpha$, for fixed $\psi$ and $\gamma$. A schematic summary is shown in Fig. 2. Additionally, we shall require the inverse maps $m^{-1}(\psi)=\varphi$ and $s^{-1}(\gamma ; \alpha)=\psi$ to exist. Explicitly, the scattering maps are

$$
\begin{aligned}
s(\psi ; \alpha) & =\psi+\alpha \equiv \gamma, \\
s^{-1}(\gamma ; \alpha) & =\gamma-\alpha \equiv \psi, \\
a(\psi, \gamma)=\gamma-\psi & \equiv \alpha .
\end{aligned}
$$

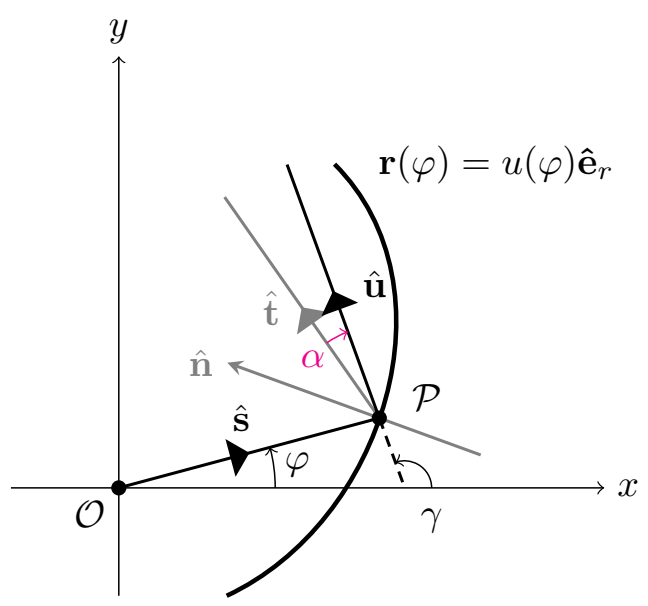

Figure 1. Cross-section of a diffuse cylindrically symmetric reflector in the plane of incidence. 


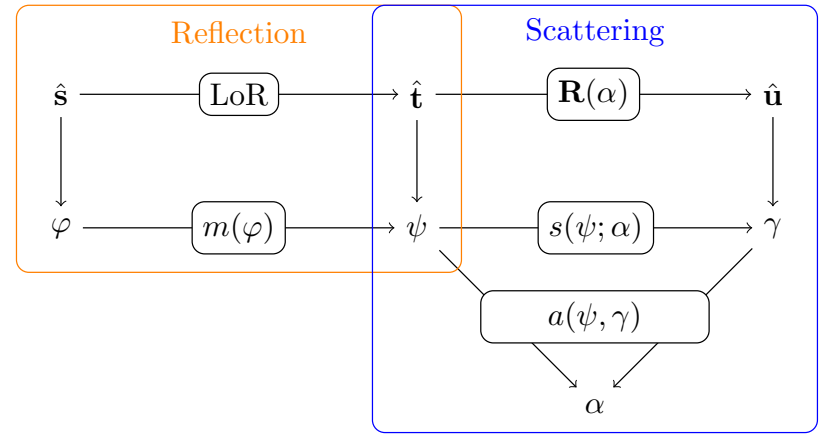

Figure 2. Mappings between unit vectors and angles.

Let $f:\left[\varphi_{1}, \varphi_{2}\right] \rightarrow \mathbb{R}^{+}$be the source distribution, $h:\left[\gamma_{1}, \gamma_{2}\right] \rightarrow \mathbb{R}^{+}$be the prescribed diffuse target distribution, and $g:\left[\psi_{1}, \psi_{2}\right] \rightarrow \mathbb{R}^{+}$be an intermediate specular target distribution $[1 \mathrm{~m} / \mathrm{rad}]$. Additionally, let $p:\left[\alpha_{1}, \alpha_{2}\right] \rightarrow$ $\mathbb{R}^{+}$, be a probability density function (PDF) representing the scattering characteristics of the surface. Since $p$ is a PDF, we have $\int_{\alpha_{1}}^{\alpha_{2}} p(\alpha) \mathrm{d} \alpha=1$. Finally, let $\rho$ : $\left[\psi_{1}, \psi_{2}\right] \times\left[\gamma_{1}, \gamma_{2}\right] \rightarrow \mathbb{R}^{+}$, with the properties

$$
\int_{\gamma_{1}}^{\gamma_{2}} \rho(\psi, \gamma) \mathrm{d} \gamma=g(\psi), \quad \int_{\psi_{1}}^{\psi_{2}} \rho(\psi, \gamma) \mathrm{d} \psi=h(\gamma),
$$

be a density of the form $\rho(\psi, \gamma)=p(a(\psi, \gamma)) g(\psi)=$ $p(\gamma-\psi) g(\psi)$, which gives the light in direction $\gamma$ when integrated with respect to $\psi$, and vice versa. Then the following convolution integral relations follow from the second relation in Eq. (3):

$$
\begin{aligned}
& h(\gamma)=\int_{\psi_{1}}^{\psi_{2}} p(\gamma-\psi) g(\psi) \mathrm{d} \psi=:(p * g)(\gamma), \\
& h(\gamma)=\int_{\alpha_{1}}^{\alpha_{2}} p(\alpha) g(\gamma-\alpha) \mathrm{d} \alpha=:(g * p)(\gamma),
\end{aligned}
$$

where we transformed the integration variable $\psi$ to $\alpha$ to reach the second relation. Physically, the density $\rho$ represents a redistribution of light in direction $\psi$ due to scattering via the scattering probability density function $p$. Note that mirrors mostly reflect in the specular direction, so that $p$ should peak for $\alpha=0$ and fall off rapidly to zero for most relevant surfaces.

\subsection{Rotationally Symmetric Reflectors}

Following an analogous procedure for rotationally symmetric problems, i.e., problems where we can consider not a translationally invariant cross-section of the reflector, but rather a rotationally invariant cross-section, the integral relations for the diffuse distribution in Eq. (4) become

$$
\tilde{h}(\gamma)=\int_{\psi_{1}}^{\psi_{2}} p(\gamma-\psi) \tilde{g}(\psi) \mathrm{d} \psi, \tilde{h}(\gamma)=\int_{\alpha_{1}}^{\alpha_{2}} p(\alpha) \tilde{g}(\gamma-\alpha) \mathrm{d} \alpha,
$$

where $\tilde{h}(\gamma):=h(\gamma) \sin (\gamma)$ and $\tilde{g}(\psi):=g(\psi) \sin (\psi)$, and where $\psi, \gamma \in[0, \pi]$ are now polar angles, making the sine

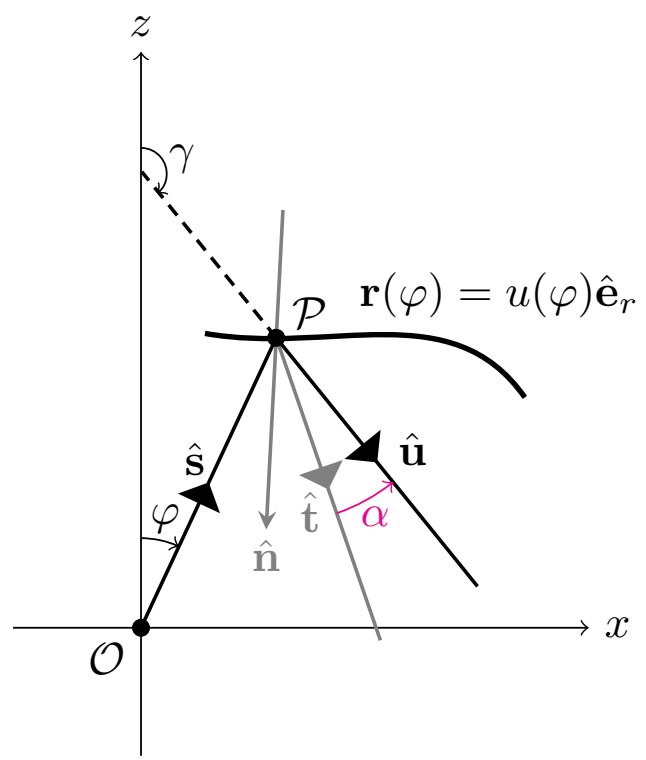

Figure 3. Cross-section of a diffuse rotationally symmetric reflector along the plane of incidence.

terms area elements in spherical coordinates. The situation is depicted in Fig. 3, where the source ray along $\hat{\mathbf{s}}$ emitted from the point source in $O$, strikes the reflector at $\mathcal{P}$, where the normal is given by $\hat{\mathbf{n}}$. The specular direction $\hat{\mathbf{t}}$ is characterised by $\psi$ (not shown), and the scattered direction $\hat{\mathbf{u}}$ is given by the angle $\gamma$. We note that the coordinate system is such that the radial unit vector $\hat{\mathbf{e}}_{r}=(\sin (\varphi), \cos (\varphi)), \varphi \in[0, \pi)$.

\section{Specular Reflector Design}

Our goal is to determine a specular reflector which transforms the given source distribution into the given specular target distribution. The approach we have chosen involves solving two ordinary differential equations - one for the radius function $u(\varphi)$ and one for the mapping $m(\varphi)$ - which together fully characterise the reflector. This is similar to the approach outlined in [2, Ch. 3.3] and [3].

\subsection{Cylindrically Symmetric Reflectors}

From the law of reflection, by computing $\hat{\mathbf{n}}$ in terms of $u$ and $u^{\prime}$, and by fixing $u\left(\varphi_{1}\right)=1$, we get the following initial value problem for the radius function $u$ :

$$
\frac{u^{\prime}(\varphi)}{u(\varphi)}=\cot \left(\frac{m(\varphi)-\varphi}{2}\right), \quad u\left(\varphi_{1}\right)=1 .
$$

There are many valid choices for the specular map $m$. Consider first a monotonically increasing $m:=m_{\mathrm{div}}$, resulting in a diverging ray-bundle. Then the following integral relation and equivalent initial value problem must hold for all $\varphi \in\left[\varphi_{1}, \varphi_{2}\right]$ :

$$
\begin{aligned}
& \int_{\varphi_{1}}^{\varphi} f(\tilde{\varphi}) \mathrm{d} \tilde{\varphi}=\int_{\psi_{1}}^{m_{\text {div }}(\varphi)} g(\tilde{\psi}) \mathrm{d} \tilde{\psi}, \\
& m_{\text {div }}^{\prime}(\varphi)=\frac{f(\varphi)}{g\left(m_{\text {div }}(\varphi)\right)}, \quad m_{\text {div }}\left(\varphi_{1}\right)=\psi_{1} .
\end{aligned}
$$


Similarly, for a monotonically decreasing $m:=m_{\text {conv }}$, yielding a converging ray-bundle,

$$
\begin{aligned}
& \int_{\varphi_{1}}^{\varphi} f(\tilde{\varphi}) \mathrm{d} \tilde{\varphi}=\int_{m_{\text {conv }}(\varphi)}^{\psi_{2}} g(\tilde{\psi}) \mathrm{d} \tilde{\psi}, \\
& m_{\text {conv }}^{\prime}(\varphi)=-\frac{f(\varphi)}{g\left(m_{\text {conv }}(\varphi)\right)}, \quad m_{\text {conv }}\left(\varphi_{1}\right)=\psi_{2} .
\end{aligned}
$$

\subsection{Rotationally Symmetric Reflectors}

In the case of rotationally symmetric reflectors, we measure $\varphi$ clockwise from the positive $z$-axis, in the plane of incidence. The reflector is now parametrised by $\mathbf{r}(\varphi)=$ $u(\varphi) \hat{\mathbf{e}}_{r}$, where $\hat{\mathbf{e}}_{r}=(\sin (\varphi), \cos (\varphi))^{\top}$ is the radial unit vector in this particular polar coordinate system. Following an analogous procedure to the one employed in the case of cylindrically symmetric reflectors, we get the following initial value problem for the monotonically increasing and decreasing maps, respectively:

$$
m_{\mathrm{div}}^{\prime}(\varphi)=\frac{\tilde{f}(\varphi)}{\tilde{g}\left(m_{\mathrm{div}}(\varphi)\right)}, \quad m_{\mathrm{div}}\left(\varphi_{1}\right)=\psi_{1},
$$

and

$$
m_{\text {conv }}^{\prime}(\varphi)=-\frac{\tilde{f}(\varphi)}{\tilde{g}\left(m_{\mathrm{conv}}(\varphi)\right)}, \quad m_{\mathrm{conv}}\left(\varphi_{1}\right)=\psi_{2} .
$$

The initial value problem for the radius function $u$ remains the same.

\section{Solution Algorithm}

The solution algorithm we propose is as follows. Given the source distribution $f$, diffuse target distribution $h$ and scattering probability density function $p$,

1. Deconvolve $g$ from Eqs. (4) for cylindrically symmetric problems, or $\tilde{g}$ from Eqs. (5) for rotationally symmetric problems. Call this deconvolved distribution $g_{\mathrm{dc}}$ or $\tilde{g}_{\mathrm{dc}}$. We used Matlab's deconvlucy.

2. Compute the reflector(s) using $f$ and $g_{\mathrm{dc}}$ or $\tilde{f}$ and $\tilde{g}_{\mathrm{dc}}$ by solving the relevant initial value problem in Eq. (7), (8), (9), or (10). We solved all of the above using Matlab's ode15s.

3. Compute the "reconvolved" diffuse distribution $h_{\mathrm{rc}}:=g_{\mathrm{dc}} * p$ or $\tilde{h}_{\mathrm{dc}}:=\tilde{g}_{\mathrm{dc}} * p$. We used Matlab's conv. This step is optional, but it will help us validate our prediction for the scattered distribution.

4. Validate the reflector(s) using raytracing.
The validation via raytracing uses our own raytracer in two dimensions, which implements scattering in accordance with our model, i.e., rotation of the reflected ray by a stochastic parameter $\alpha$ sampled from $p$.

\section{Results}

The results for a pair of cylindrically symmetric reflectors is shown in Fig. 4, where $f$ is a block function, $p$ is a Gaussian and the raytraced diffuse distribution $h_{\mathrm{rc}}^{*}$ is very close to our prescribed $h$. The raytraced reflector is $m_{\text {conv }}$ and the root mean squre (RMS) error $\varepsilon\left(h, h^{*}\right)=$ $\sqrt{\frac{1}{N} \sum_{n=1}^{N}\left|h_{n}-h_{n}^{*}\right|^{2}}$ goes to zero $\propto N_{\mathrm{r}}^{-1 / 2}$, where $N_{\mathrm{r}}$ is the number of rays traced and the sum runs over the number of collection bins $N$, as expected from Monte Carlo raytracing [4, p. 9]. Turning to Fig. 5, we see an example of a rotationally symmetric reflector. For this example, we do not know $\tilde{g}$ exact, but we show $\tilde{g}_{\mathrm{dc}}$, which is convolved with $p$ - this time a Lorentzian (Cauchy) distribution to form $h_{\mathrm{rc}}$, representing the predicted diffuse light distribution. The raytraced reflector is once more $m_{\text {conv }}$, which is also shown in three dimensions for the sake of completion. The convergence of the raytraced distribution follows the expected $N_{\mathrm{r}}^{-1 / 2}$ behaviour.

\section{Conclusions}

Our novel modelling approach to include surface scattering in the design of reflectors as part of optical systems reduces to a convolution problem for cylindrically and rotationally symmetric problems. Solving the convolution integral yields a specular target distribution which can be used in the mature framework of specular reflector design.

Funding: This work was partially supported by the Nederlandse Organisatie voor Wetenschappelijk Onderzoek through grant P15-36.

\section{References}

[1] L.B. Romijn, J.H.M. ten Thije Boonkkamp, W.L. IJzerman, Journal of Computational Physics 408 (2020)

[2] M.J.J.J.B. Maes, Ph.D. thesis, University of Amsterdam (1997)

[3] V.C.E. Kronberg, M.J.H. Anthonissen, J.H.M. ten Thije Boonkkamp, W.L. IJzerman, Journal of the Optical Society of America A (2021), submitted

[4] C. Filosa, Ph.D. thesis, Eindhoven University of Technology (2018) 

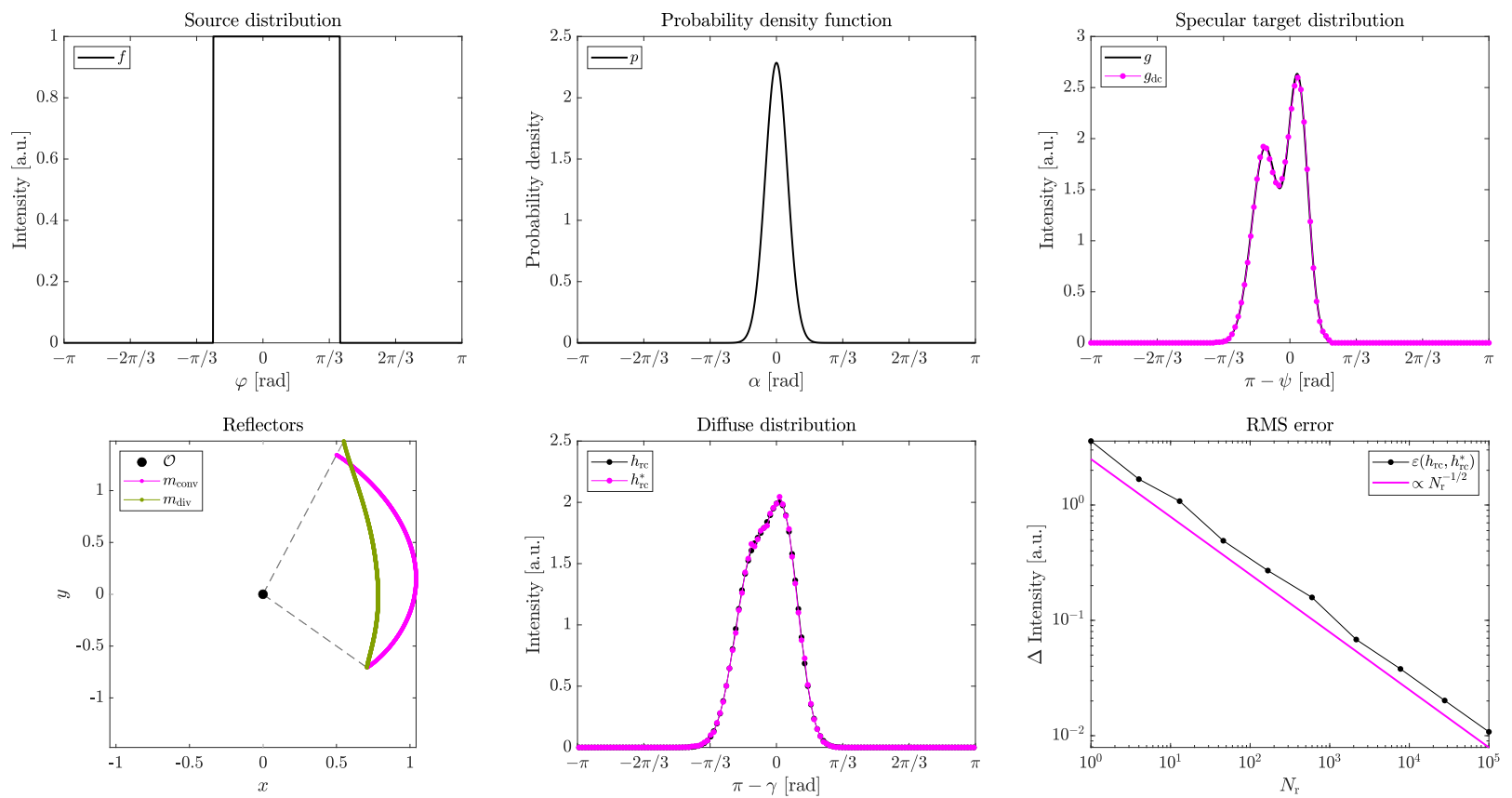

Figure 4. Example of cylindrically symmetric reflector design procedure. The prescribed diffuse distribution $h$ is achieved, and the raytraced distribution converges with the expected square-root behaviour.
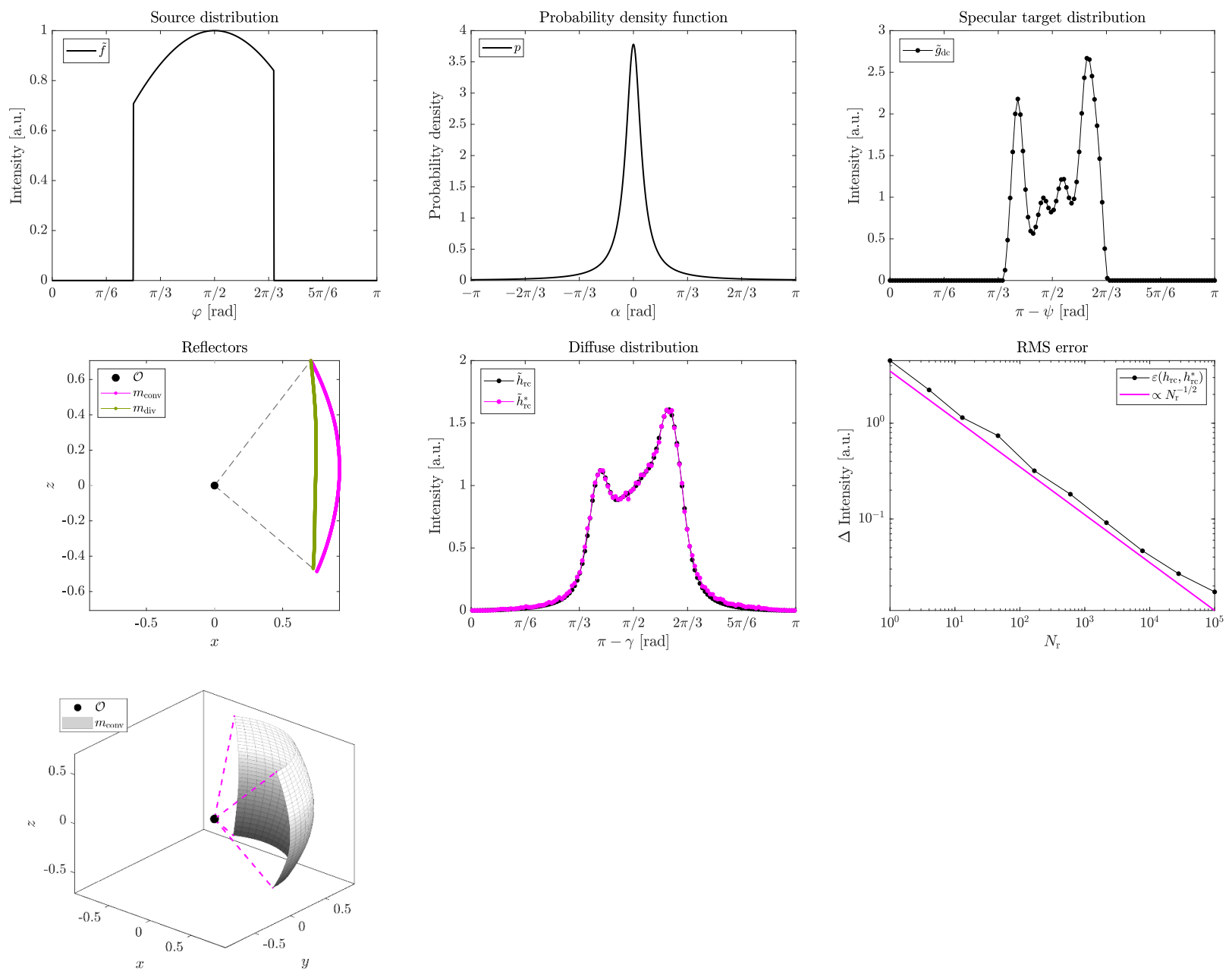

Figure 5. Example of rotationally symmetric reflector design procedure. The prescribed diffuse distribution $\tilde{h}_{\mathrm{rc}}$ is achieved, and the raytraced distribution (roughly) converges with the expected square-root behaviour. The 3D representation of $m_{\text {conv }}$ is included for the sake of completion. 